\title{
Influencia del desempeño docente en el logro del perfil de egreso en estudiantes universitarios
}

\author{
Influence of teaching performance on the achievement of the graduation profile in university students
}

Influência do desempenho docente no alcance do perfil de graduação em estudantes universitários

Wido Willam Condori Castillo

widocondori@unap.edu.pe

https://orcid.org/0000-0001-6569-9148

Universidad Nacional del Altiplano, Puno-Perú

Percy Samuel Yábar Miranda

p.yabar@unap.edu.pe

https://orcid.org/0000-002-3182-9802

Universidad Nacional del Altiplano, Puno-Perú
Fredy Sosa Gutiérrez

fredysosa@unap.edu.pe

https://orcid.org/0000-0001-6473-3877

Universidad Nacional del Altiplano, Puno-Perú

Patricia Quispe Ramos

patyq53way@gmail.com

https://orcid.org/0000-0001-9580-9475

Universidad Nacional del Altiplano, Puno-Perú

Artículo recibido 6 de septiembre 2021, arbitrado y aceptado 13 de octubre 2021 y publicado 20 de diciembre 2021

\section{RESUMEN}

La investigación determina la incidencia entre el desempeño docente y el logro del perfil del egreso, al mismo tiempo busca identificar la prevalencia de las dimensiones facilitador, investigador y promotor del logro del perfil del egreso. El trabajo corresponde al enfoque cuantitativo de tipo no experimental, de diseño correlacional y explicativo, teniendo como población a estudiantes del $\mathrm{X}$ semestre 2018-II, a quienes se administró un cuestionario (desempeño docente), prueba de conocimientos, rúbricas y escalas de estimación (logro del perfil del egreso). Los resultados obtenidos del coeficiente de correlación de Rho Spearman demuestran una asociación positiva muy alta $(\mathrm{r}=$ 0.849 ) entre las variables, además se tiene un coeficiente de determinación R2 $=0.746$ que nos da a conocer que aproximadamente el $74.6 \%$ de la variación de la variable del perfil de logro del egresado puede ser explicada por la variación de la variable desempeño docente.

Palabras clave: Competencia; Currículo; Desempeño docente; Evaluación; Perfil del egreso, Programa de estudio

\section{ABSTRACT}

The research determines the incidence between teaching performance and the achievement of the graduation profile, at the same time it seeks to identify the prevalence of the facilitator, researcher and promoter dimensions of the achievement of the graduation profile. The work corresponds to a nonexperimental quantitative approach, with a correlational and explanatory design, having as a population student from the $\mathrm{X}$ semester 2018-II, who were administered a questionnaire (teaching performance) and knowledge test, rubrics and estimation scales (achievement of the graduation profile). The results obtained from the Rho Spearman correlation coefficient show a very high positive association $(r=0.849)$ between the variables, in addition there is a determination coefficient $\mathrm{R} 2=0.746$ that reveals that approximately $74.6 \%$ of the variation of the variable of the graduate's achievement profile can be explained by the variation of the teacher performance variable.

Key words: Competence; Curriculum; Teaching performance; Evaluation; Graduation Profile, Study program

\section{RESUMO}

A pesquisa determina a incidência entre o desempenho docente e o alcance do perfil de graduação, ao mesmo tempo em que busca identificar a prevalência das dimensões facilitadora, pesquisadora e promotora do alcance do perfil de graduação. $\mathrm{O}$ trabalho corresponde a uma abordagem quantitativa não experimental, com um desenho correlacional e explicativo, tendo como população alunos do X semestre 2018-II, aos quais foi aplicado um questionário (desempenho docente) e teste de conhecimentos, rubricas e escalas de estimativa (aproveitamento do perfil de graduação). Os resultados obtidos a partir do coeficiente de correlação de Rho Spearman mostram uma associação positiva muito elevada $(r=0,849)$ entre as variáveis, além disso há um coeficiente de determinação $\mathrm{R} 2=0,746$ que revela que aproximadamente $74,6 \%$ da variação da variável do graduado perfil de desempenho pode ser explicado pela variação da variável de desempenho do professor.

Palavras-chave: Competência; Currículo; Desempenho docente; Avaliação; Perfil de graduação, Programa de estudos 


\section{INTRODUCCIÓN}

Los perfiles de egreso es el conjunto de competencias en el marco de los dominios profesionales propios de la especialidad, los cuales tienen que ser desarrollados a lolargo dela formación profesional. La evaluación de las competencias y por competencias es considerada como un proceso de retroalimentación, determinación de idoneidad y certificación de los aprendizajes de los estudiantes (Zabalza, 2003) por lo que su implementación es poco esclarecida al término de la formación en el Programa de Estudios de educación Primaria de la Universidad Nacional del Altiplano-Puno. El desempeño docente se ve, en cierto modo, reflejado en la formación de los estudiantes; es decir en el logro de las competencias previstas en el currículo universitario, motivo por el que la investigación aborda el problema referido a ¿cuál es la incidencia del desempeño docente en el logro del perfil de egreso?, al mismo tiempo pretende explicar ¿de qué manera incide el desempeño docente en las dimensiones facilitador, investigador y promotor delo logro del perfil de egreso?

Barrientos (2013) considera que el docente universitario es la persona que se dedica a cultivar $\mathrm{y}$ transmitir saberes, realiza investigaciones educativas o en su campo profesional, y gestiona la responsabilidad social; por lo que, según Benito y Cruz (2005) debe contar con competencias referidas al conocimiento disciplinar, innovación, dominio de la gestión del currículo, motivación para el aprendizaje, habilidades comunicativas, aprendizaje colaborativo y comprometido con la ética de la profesión docente.

Zabalza (2003) considera tres dimensiones del docente universitario profesional, personal y laboral. En esta misma línea, Echeverría (2002) indica que para desempeñar eficientemente una profesión es necesario saber los conocimientos requeridos por la misma tales como el componente técnico, personal y participativo. De otra parte, Tobón (2005) considera que, desde el enfoque de la formación basada en competencias, el énfasis no está en los estudiantes, ni tampoco en los docentes, sino en la relación intersistémica de ambos. A esto, Quesada (2001) señala que, el énfasis está en los estudiantes, buscando que estos aprendan estrategias que les permitan autorregular su aprendizaje.

Según Tobón (2015) hay dos conceptos da la docencia estratégica: conocimiento y autorregulación. Conocer implica para el docente hacer parte del aprendizaje de los estudiantes, involucrándose en sus metas y colocándose en su lugar sin perder el propio rol de maestro, y la autorregulación tiene tres acciones: planear, monitorear y valorar. El valorar implica la evaluación. En este sentido, Mateo (2000) señala que existe, sin duda, un renovado interés por el papel que juega la evaluación del docente en la mejora de la universidad. Lourerio y Miguéz (2006) señalan que la evaluación docente debe ser un insumo para tomar decisiones orientadas a mejorar el proceso de enseñanza y la identificación de necesidades de formación, desarrollando las estrategias más adecuadas para satisfacerlas.

En este sentido, la evaluación del docente universitario es entendida como una tarea multidimensional y compleja, que debe realizarse a partir de información procedente de diversas fuentes, empleando un enfoque multiestratégico (Mateo et al., 1996; Muñiz, García y Virgos, 1991; Muñoz, Ríos y Abalde, 2002). Es importante considerar que, en una evaluación integral se encuentran las opiniones de los estudiantes, la 
autoevaluación del docente, la evaluación por parte del superior jerárquico, la evaluación de sus pares y la observación metodológica (Muñoz, Ríos y Abalde, 2002). En el marco de la evaluación, Valdéz (2000) plantea modelos de la evaluación del desempeño docente, centrado en el perfil del docente, en los resultados obtenidos, en el comportamiento del docente en el aula y de la práctica reflexiva.

En el marco de las funciones de la evaluación del desempeño docente, algunos estudios como de Carrizales y Rodríguez (1999); Cruz (2007); Stiggins y Duke (1988); Rebolledo y Ayala (2006) muestran una serie de coincidencias en determinar las funciones de la evaluación al docente, considerando que una buena evaluación al desempeño docente debe cumplir las siguientes funciones: diagnóstica, instructiva, educativa, desarrolladora. Dentro de estas funciones, la competencia y desempeño docente, Le Boterf (2001) define el término competencia como la capacidad de movilizar y aplicar correctamente en un entorno laboral determinado, recursos propios (habilidades, conocimientos y actitudes) con fines de lograr un resultado definido (Producto o servicio), cumpliendo estándares o criterios de calidad.

Por su parte, el Sistema Nacional de Evaluación y Acreditación de la Calidad Educativa (Sineace, 2016) en la dimensión de Gestión Docente del modelo de acreditación que involucra estándares $14,15,16$ y 17 , se pretende que los programas de estudio implementen mecanismos de selección, evaluación, capacitación y perfeccionamiento; plana docente adecuada; reconocimiento de las actividades de labor docente; y el plan de desarrollo académico del docente, se busca además, que el programa de estudios tenga mecanismos que evalúan el desempeño docente con la finalidad de identificar necesidades de capacitación y perfeccionamiento o separación.

La evaluación del desempeño docente en la Universidad Nacional del altiplano de Puno se rige por su reglamento, aprobado por Resolución Rectoral $N^{\circ}$ 1243-2018-R-UNA, que rige a partir del año 2018. Al mismo tiempo, la evaluación a los docentes es obligatoria y semestral, constituye un proceso sistemático de recopilación de información que, luego de ser procesada, sirve para tomar decisiones orientadas a la mejora continua del desempeño profesional, académico, de investigación y de responsabilidad social de los docentes. En la evaluación del desempeño docente, participan las autoridades como el Decano, Director de Departamento, Director de la Escuela Profesional, Coordinadores de Investigación, Coordinadores de Responsabilidad Social, Coordinadores de Tutoría y los estudiantes, matriculados en cada componente curricular y que asisten regularmente a las sesiones de aprendizaje (Universidad Nacional del Altiplano, 2018).

Irigoin (2006), justifica que una carrera universitaria de pregrado necesita tener un perfil de egreso. Es decir, en términos simples, la declaración pública de aquellas competencias que un estudiante debe tener para que la universidad le otorgue un determinado grado o título al egresar de su carrera, y para los cuales se espera lograr. El perfil de egreso debe ser el punto de convergencia entre los intereses $\mathrm{y}$ avances del mundo sociocultural y laboral, de las profesiones, las disciplinas, de los estudiantes, y de la universidad a través de sus principios y valores institucionales (Tobón, 2006). 
En el marco del perfil del egresado del programa de estudios de Educación Primaria, el perfil académico-profesional de competencias, es el perfil ideal de salida o del egresado. Constituye la descripción de los rasgos que deben caracterizar a un estudiante al culminar un proceso educativo. Describe las características del egresado en función de las actividades que como futuro profesional estará en condiciones de realizar en el campo educativo. De este modo el perfil del egresado está organizado por funciones (función facilitadora, función investigadora y función promotora) y saberes (saber conocer, saber hacer, saber ser y saber convivir).

Según las áreas de formación académica profesional, se organizan los contenidos que permitirán el desarrollo de actividades cognitivas e instrumentales a fin de lograr los objetivos de formación profesional. Las áreas están en relación con las funciones del perfil. De acuerdo a la ley universitaria No 30220 se organizan en área general, área específica y área especializada. En esta línea es preciso considerar a Tobón (2017) quien considera el enfoque socioformativo $y$ el pensamiento complejo enfatiza en cómo cambiar la educación desde el cambio del pensamiento de las personas responsables de ella a través de la investigaciónacción, teniendo en cuenta la persona humana cómo un todo, una de cuyas dimensiones son las competencias. Tobón et al., (2015) consideran que la socioformación implica que los ciudadanos sean capaces de resolver problemas del contexto, gestionando el conocimiento en diferentes fuentes con un sentido crítico y trabajando de manera colaborativa. De hecho, estas implicancias nos conducirían al logro de perfil de egreso de los estudiantes. El logro de las competencias requeridas, obviamente exige de la evaluación formativa, lo que para Huanca (2017), se debe presentar un análisis de saberes previos, abordaje de intereses de los estudiantes, autorregulación del estudiante, evaluación mediante situaciones con sentido o dinámicas. Estas actividades contribuirían al logro del perfil de egreso. El papel del docente es acompañar y orientar a los estudiantes a que se autoevalúen y mejoren (Tobón, 2015).

La búsqueda del logro del perfil de egreso, está vinculada a la evaluación Gutiérrez (2018) busca garantizar el desarrollo midiendo el nivel de desempeño y teniendo en cuenta habilidades como el uso y contextualización del conocimiento para generar soluciones y niveles de desempeño para su medición. Es por ello que el enfoque socioformativo, contribuye al desarrollo, porque implica orientar la formación a la mejora de condiciones de vida (Tobón, 2018). La evaluación en la formación por competencias se escalona en un continuo de aprendizajes que nos lleva al logro de perfil. Fernández (2010) toma en cuenta algunas consideraciones referidas a que las evaluaciones requieren instrumentos complejos y variados que deben de constituir una oportunidad de aprendizaje que involucre a diferentes agentes (docentes, estudiantes y otros), proporcionando información sobre el progreso y desarrollo de la competencia, así como, sugiriendo caminos de mejora, que permitan a los estudiantes conocer su nivel de competencias. En este escenario, Tobón (2015) sostiene que el papel del docente es acompañar y orientar a los estudiantes a que se autoevalúen y mejoren. Tobón (2018) y Huanca (2017) coinciden en indicar que la evaluación desde el enfoque socioformativo 
se orienta directamente a la actividad y no a los objetivos, busca el desarrollo máximo de las competencias en base a problemas del contexto y el análisis de evidencias para mejorar continuamente el talento humano.

El objetivo que persigue la investigación es determinar la incidencia del desempeño docente en el logro del perfil de egreso, en los estudiantes del Programa de Estudios de Educación Primaria de la Universidad Nacional del Altiplano-Puno, 2018 II. Esclarecer la vinculación y su incidencia entre las variables permitirá reflexionar y mejorar los procesos de enseñanza y aprendizaje en el ejercicio del desempeño docente en la educación superior, con el propósito de garantizar el logro del perfil del egreso de estudiantes universitarios.

\section{MÉTODO}

El estudio se enmarcó en el enfoque cualitativo de tipo no experimental y diseño correlacional, empírico-explicativo y la regresión lineal simple (Hernández y Mendoza, 2018).

Los esquemas de los diseños aplicados son:

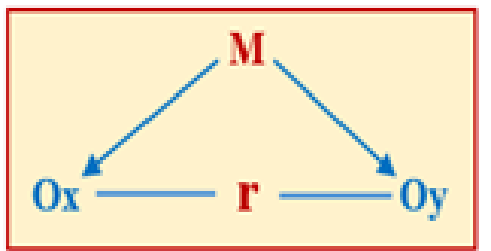

\section{Donde:}

$\mathrm{M}=$ Representa la muestra de estudio

$\mathrm{Ox}=$ Desempeño Docente

Oy $=$ Logro del perfil del egresado

$\mathrm{r} \quad=$ Indica el grado de relación entre ambas variables.

Correlacional. Permitió determinar el grado de correlación que existe entre las variables del desempeño del docente y el logro del perfil de egreso, así como en su funcionalidad como facilitador, investigador y promotor como futuro docente.
Su diseño es:

$$
\mathbf{y}=(\mathbf{x})
$$

\section{Donde:}

$\mathrm{x}=$ Desempeño Docente

$\mathrm{y}=$ Logro del perfil de egreso

$f=$ Función de dependencia entre ambas variables.

Empírico-explicativo (inferencial): Busca explicar cómo incide el desempeño docente en el logro del perfil del egresado, así como en su funcionalidad como facilitador, investigador y promotor.

Regresión Lineal Simple (RLS). Técnica de análisis estadístico utilizada para estimar el efecto de una variable (independientes/predictores) en otra variable cuantitativa (dependiente/predicha/ respuesta). El objetivo de la regresión es: Determinar cómo incide X (Desempeño docente) en Y (Perfil de logro del Egresado).

En el modelo de regresión lineal simple se explicó la relación que existe entre la variable respuesta $\mathrm{Y}$ (perfil de logro del egresado) y una única variable explicativa $X$ (Desempeño docente).

El modelo de la recta es:

$$
Y=\beta_{1}+\beta_{2} X
$$

\section{Donde:}

$\mathrm{Y}=$ Perfil de logro del egresado

$\beta_{1}=$ Constante $y / o$ intercepto

$\beta_{2}=$ Coeficiente del modelo (pendiente)

$\mathrm{X}=$ Desempeño docente

Según su alcance temporal, es una investigación transversal, puesto que se recolectó los datos en un tiempo único, cuyo propósito fue describir las variables y analizar su incidencia e interrelación en un momento dado. 
La muestra estuvo conformada por los estudiantes matriculados en el $\mathrm{X}$ semestre del año académico 2018-II del Programa de Estudios de Educación Primaria de la Universidad Nacional del Altiplano, Puno. Se eligió de manera intencional basado en criterios técnicos, considerando que la Coordinación Académica de la Facultad de Ciencias de la Educación informó que los estudiantes aprobados y expeditos para ser egresados del programa son 21 estudiantes a quienes se administró los instrumentos de recolección de datos.

Las técnicas implementadas durante la recolección de la información son la encuesta, examen, observación sistemática y escala de estimación. Los instrumentos utilizados son el cuestionario para recabar información de la variable desempeño docente, y la prueba de conocimientos, rúbricas y escalas numéricas los cuales garantizaron recabar información de la variable logro del perfil del egreso. Los instrumentos han sido validados por expertos quienes emiten opinión favorable para su aplicación.
RESULTADOS

En este apartado se muestran los resultados obtenidos de las variables desempeño docente y el logro del perfil del egreso, incluyendo resultados de las dimensiones planteadas. Consecuentemente, se muestra las correlaciones entre ambas variables y las incidencias del desempeño docente en el logro del perfil del egreso y sus dimensiones facilitador, investigador y promotor.

\section{Evaluación del desempeño docente}

Existe un renovado interés por el papel que juega la evaluación del desempeño docente en la mejora de la universidad, dado que el docente demuestre dominio de conocimientos y procesos del componente curricular que está a su cargo, desarrolla actividades de enseñanza y aprendizaje donde los estudiantes participan activamente, utiliza adecuadamente procesos metodológicos, material didáctico, bibliografía actualizada y otros que contribuyen a un mejor aprendizaje.

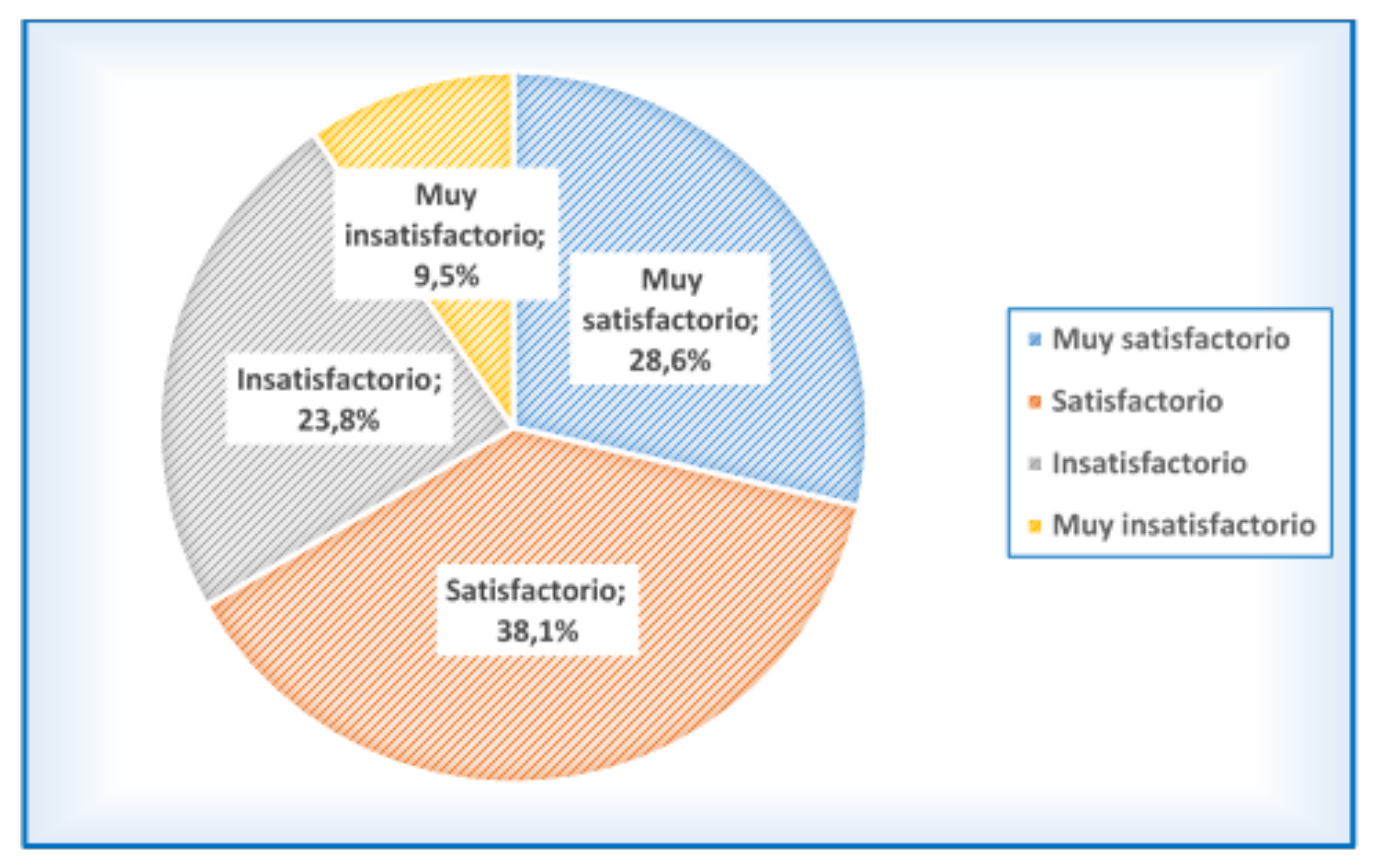

Figura 1. Evaluación del Desempeño del docente según los egresados. 
Los resultados que se presentan en la Figura 1 dan conocer que la mayoría de los estudiantes están de Muy satisfechos y satisfechos con la labor que realiza los docentes, es decir, que manejan las competencias de un saber hacer o un saber actuar en forma responsable y validada en un contexto profesional, combinando y movilizando recursos necesarios (conocimientos, habilidades, actitudes) para lograr un resultado cumpliendo estándares o criterios de calidad esperados por los egresados. Respecto al resultado, podemos vincularlo a Feeney (2001), que afirma que el propósito fundamental del sistema de evaluación, es el de mejorar la calidad del desempeño docente, que deben ser: Estimular y favorecer el interés por el desarrollo profesional de docentes, desarrollo profesional, mejorar su conocimiento y capacidades, contribuir al mejoramiento de la gestión, fortalecer la formación integral de los educandos.

\section{Logro del perfil de egreso}

Viene a ser la declaración pública de aquellas competencias que un estudiante debe tener para que la universidad le otorgue un determinado grado al egresar de su carrera, y para los cuales se espera lograr. Al mismo tiempo es un proceso interactivo que permite la valoración sobre el nivel de logro del aprendizaje alcanzado por el estudiante, en el desarrollo de las competencias, con el propósito de tomar decisiones que conlleven a la mejora de los procesos de aprendizaje y enseñanza.

La Figura 2 permite identificar que la mayoría de los egresados del Programa de estudios de educación Primaria se ubican en la escala de valoración: logro aceptable con el $42.9 \%$ de los egresados y solo el $14.3 \%$ en logro eficaz estos datos representan un total 12 egresados de 21 que logran desarrollar las competencias de las funciones de facilitador, investigador y promotor.

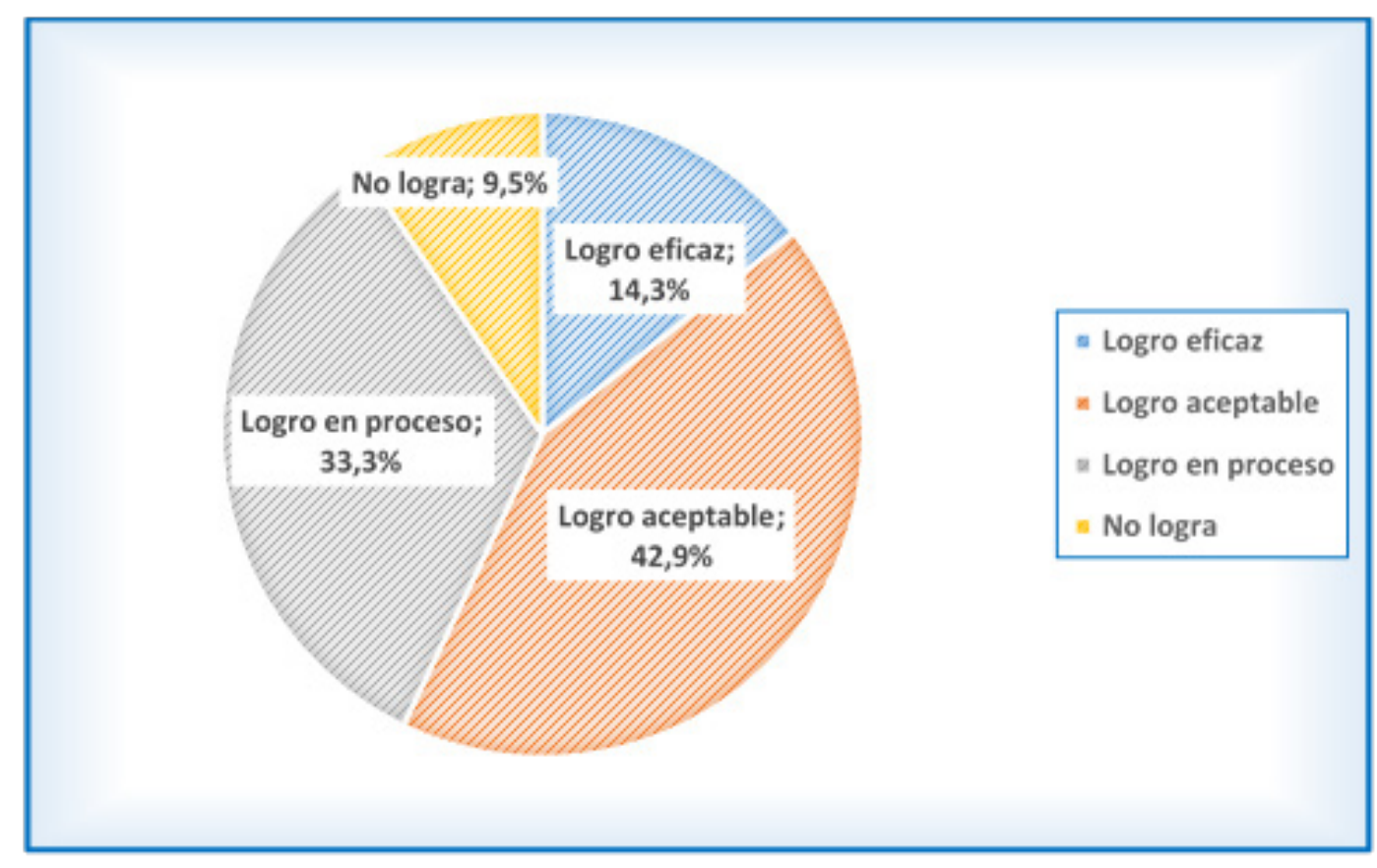

Figura 2. Distribución porcentual del logro del perfil de egreso. 
Resultados descriptivos de las funciones facilitador, investigador y promotor del logro del perfil de egreso

En la Figura 3 se puede observar que los egresados desarrollan las competencias de las funciones del logro del perfil de egreso de manera aceptable puesto que en la función de facilitador y promotor se ubicó al 43\%, en la función de investigador se encontraron al $45 \%$. Aquí se debe resaltar que buena parte de los egresados también se ubican en la escala de logro en proceso, reconocido como regular, lo que significa que los egresados muestran algunas deficiencias en el desarrollo de las competencias, los cuales deben ser mejorados en el campo de la práctica profesional.

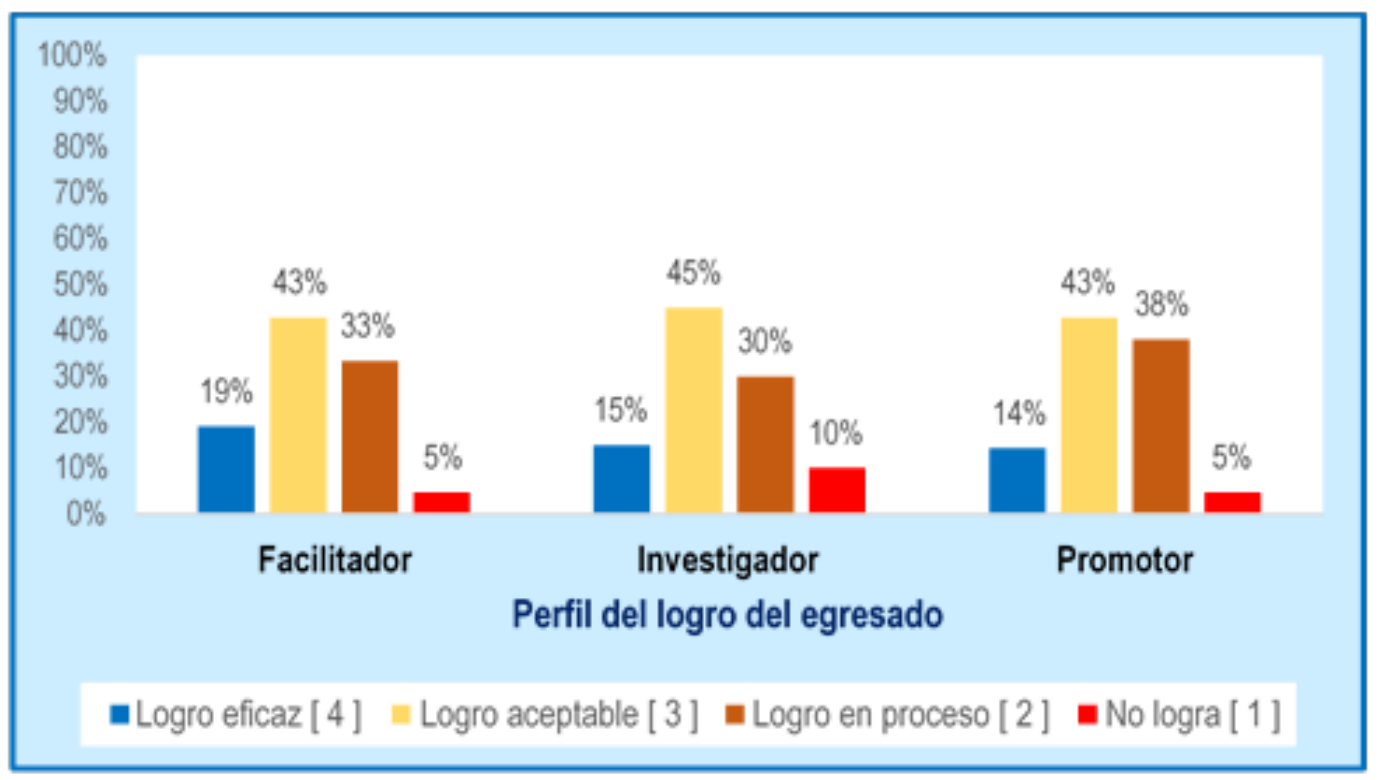

Figura 3. Distribución porcentual de las funciones del logro del perfil de egreso. Fuente: Base de datos de la investigación.

Correlación e influencia del desempeño docente y el perfil de logro del egreso

\section{Regresión Lineal Simple (RLS)}

Modelo de RLS del logro del perfil de egreso y el desempeño docente

La estimación del modelo RLS F $(1,19)=45,05$ muestran que los coeficientes son conjuntamente significativos para explicar la probabilidad de mejorar el puntaje del logro del perfil de egreso, la Prob $>\mathrm{F}=0,00$ indica que la hipotesis nula de todos los parametros del modelo (coeficientes) son iguales a cero, y el R-squared nos da conocer que aproximadamente el 70,3\% de la variación de la variable del logro del perfil de egreso puede ser explicada por la variación de la variable desempeño docente en el modelo, asimismo se cumple con los signos esperados del modelo (Tabla 2).

Modelo de la recta: $\quad Y=\beta_{1}+\beta_{2} X+\varepsilon$ 
Tabla 1. Modelo de RLS del logro del perfil de egreso y el desempeño docente.

\begin{tabular}{lrrrr}
\hline \multicolumn{1}{c}{ Variable } & Coeficiente & Desviación estándar & t calculada & $\mathbf{P}>|\mathbf{t}|$ \\
\hline Desempeño docente & 1,235192 & 0,1840395 & 6,71 & 0,000 \\
_cons & 8,608014 & 5,423183 & & \\
Obs & 21 & & & \\
F(1, 19) & 45,05 & & \\
Prob $>$ F & 0,0000 & & \\
R-squared & 0,7033 & & \\
Adj R-squared & 0,6877 & & \\
\hline
\end{tabular}

Ecuación de la recta:

$$
\text { Logegres }=8,608+1,23 * \text { desdoc }
$$

La ecuación de la recta de perfil de logro del egresado; permite determinar que el aumento de una unidad en el puntaje en el desempeño docente, el puntaje de logro del egresado aumentara en 1,23 puntos manteniendose constante las demas variables. Por tanto se prueba que el desempeño docente incide en el perfil de logro del egresado del Programa de Educación Primaria de la UNAP.

Linea de estimación de la regresión del logro del perfil de egreso y el desempeño docente
La linea de la tendencia de datos del modelo de regresión, permirte identificar la linea estimada o linea ajustada entre el puntaje logrado del perfil de egreso que esta determinada por el puntaje obtenido del desempeño del docente del Programa de Educación Primaria de la Facultad de Ciencias de la Educación de la UNAP. Asimismo, se afirma que existe una relación directa y positiva por el sentido de la linea estimada, dando a conocer que mientras aumente el puntaje obtenido de la variable del desempeño del docente aumentara el puntaje logrado de la variable del logro del perfil de egreso (Figura 4).

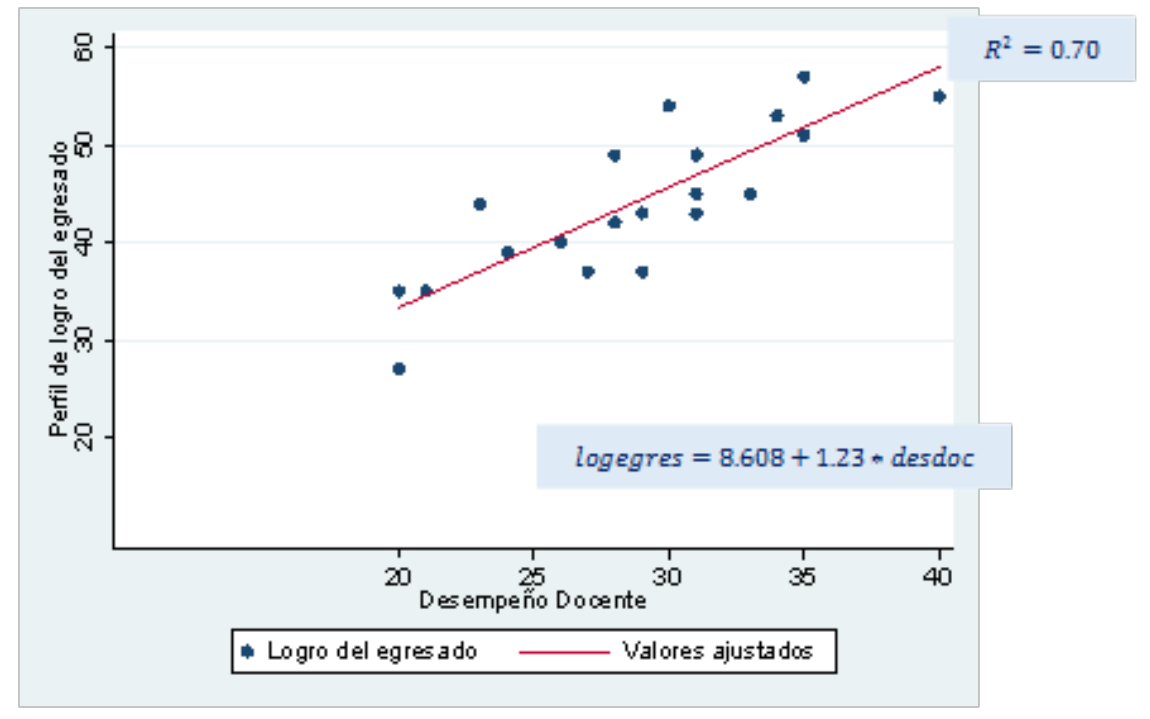

Figura 4. Línea de estimación del logro del perfil de egreso y el desempeño docente. Fuente: Base de datos de los puntajes del desempeño docente y el logro del perfil de egreso. 
Linea de estimación de la regresión de las funciones de facilitador, investigador $y$ promotor su relación con el desempeño docente

La linea de la tendencia de datos del modelo de regresión, permirte identificar la linea estimada o linea ajustada entre el puntaje logrado del logro del perfil de egreso en sus funciones de facilitador, investigador y promotor que esta determinada por el puntaje obtenido del desempeño del docente del Programa de Educación Primaria de la Facultad de Ciencias de la Educación de la UNAP (Figura 5).
La figura muestra que existe una relación directa y positiva por el sentido de la linea estimada, dando a conocer que mientras aumente el puntaje obtenido de la variable del desempeño del docente aumentara el puntaje de la función de facilitador del egresado..
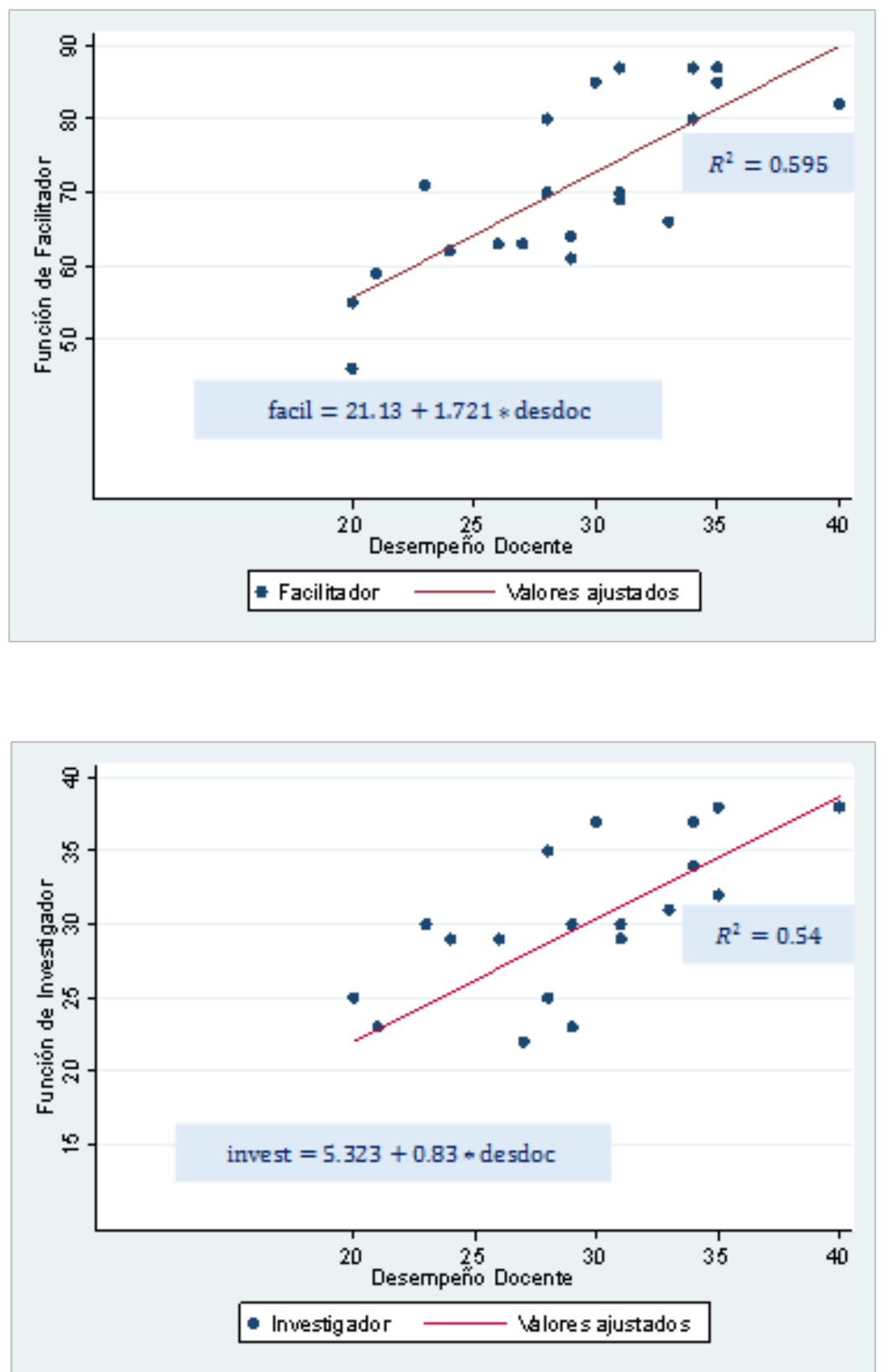

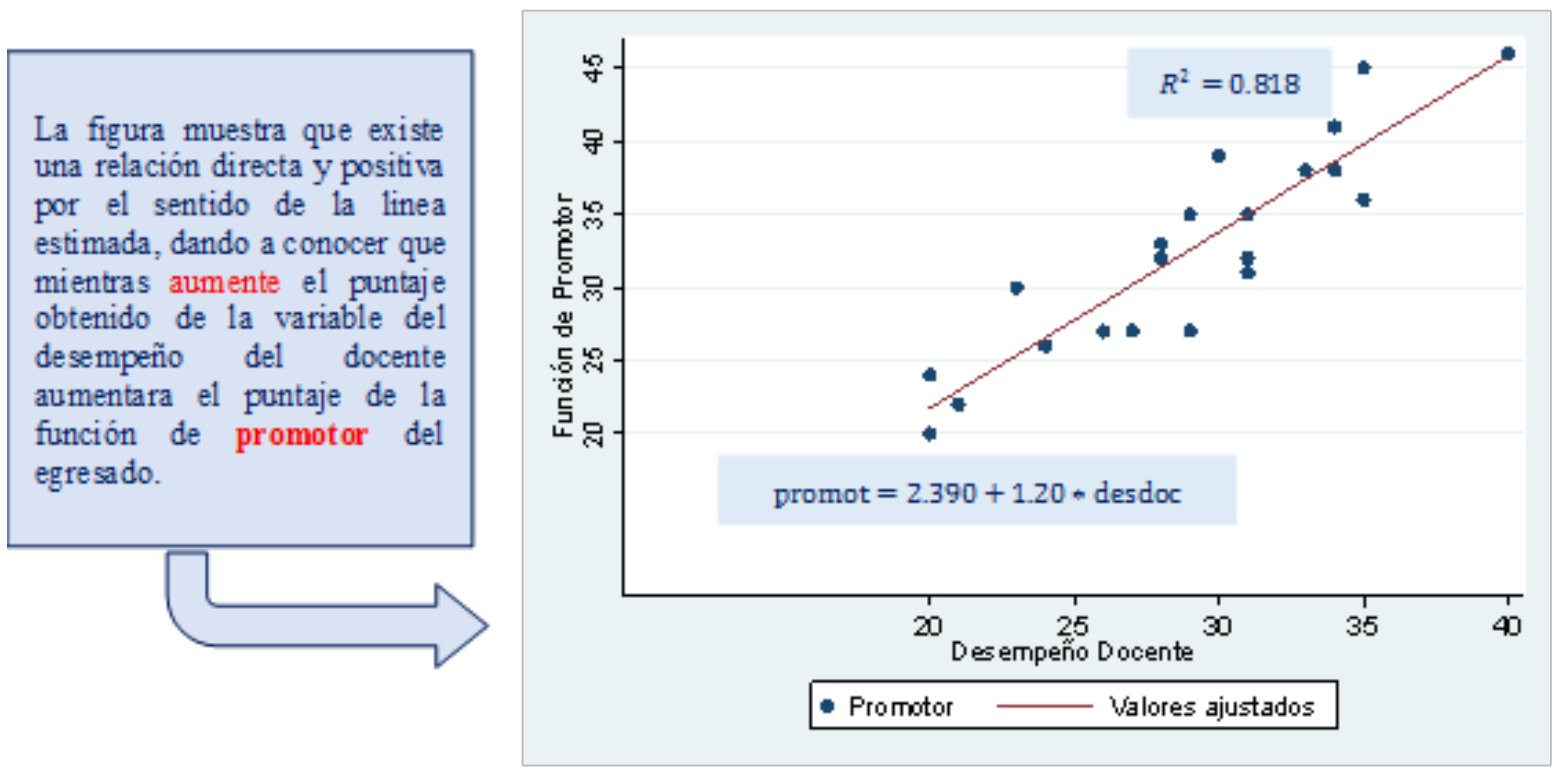

Figura 5. Línea de estimación de las funciones del egresado y el desempeño docente. Fuente: Base de datos de los puntajes del desempeño docente y el logro del perfil de egreso.

Prueba de hipótesis estadística de Spearman para el objetivo general

Se utilizó el estadístico de Prueba de Spearman puesto que los datos recolectados de los instrumentos son numerales y ordinales.

\section{Estadístico de prueba del desempeño docente y el logro del perfil de egreso}

\section{a) Planteamiento de la Hipótesis:}

$\mathrm{H}_{0}$ : No existe correlación entre los puntajes obtenidos del desempeño del docente y los puntajes hallados del logro del perfil de egreso del semestre académico 2018 - II, del Programa de Educación Primaria de la Universidad Nacional del Altiplano - Puno.
$\mathrm{H}_{1}$ : Existe correlación entre los puntajes obtenidos del desempeño del docente y los puntajes hallados del logro del perfil de egreso del semestre académico 2018 - II, del Programa de Educación Primaria de la Universidad Nacional del Altiplano - Puno.

b) Establecer un nivel de significancia $\alpha=0,05=5 \%$ margen de error.

c) Estadístico de prueba y toma de decisión Se asumió la correlación Spearman. Valor de "Rho" Calculado y Valor de P 
Tabla 2. Estadístico de prueba del desempeño docente y el logro del perfil de egreso.

\begin{tabular}{llrr}
\hline & & Desempeño docente & Perfil de logro del egresado \\
\hline & Coeficiente de correlación & 1.000 &, $\mathbf{8 4 9}^{* *}$ \\
Rho de Spearman & Sig. (bilateral) & 21 & .000 \\
\hline
\end{tabular}

\section{d) Interpretación.}

El valor de $\mathrm{P}=0.000$ está por debajo del valor de significancia es igual a 0.05 , por tanto, se acepta la hipótesis alterna $\left(\mathrm{H}_{1}\right)$ que afirma que existe correlación entre los puntajes obtenidos del desempeño del docente y los puntajes hallados del logro del perfil de egreso del semestre académico 2018 - II, del Programa de Educación Primaria de la Universidad Nacional del Altiplano - Puno.

\section{Estadístico de prueba del desempeño docente y las funciones de facilitador, investigador y promotor del perfil de egreso}

\section{a) Planteamiento de la Hipótesis:}

$\mathrm{H}_{0}$ : No existe correlación entre los puntajes obtenidos del desempeño del docente $y$ los puntajes de las funciones de facilitador, investigador y promotor del logro del perfil de egreso del semestre académico 2018 - II, del Programa de Educación Primaria de la Universidad Nacional del Altiplano - Puno.

$\mathrm{H}_{1}$ : Existe correlación entre los puntajes obtenidos del desempeño del docente y los puntajes de las funciones de facilitador, investigador $y$ promotor del logro del perfil de los egresados del semestre académico 2018 - II, del Programa de Educación Primaria de la Universidad Nacional del Altiplano - Puno.

\section{b) Establecer un nivel de significancia}

$$
\alpha=0,05=5 \% \text { margen de error. }
$$

\section{c) Estadístico de prueba y toma de decisión}

Se asumió la correlación Spearman.

Valor de "Rho" Calculado y Valor de P

Tabla 3. Estadístico de prueba del desempeño docente y las funciones de facilitador, investigador y promotor del perfil de egreso.

Desempeño docente 


\section{Desempeño docente Función de promotor}

\section{d) Interpretación}

El valor de $\mathrm{P}=0.000$ está por debajo del valor de significancia es igual a 0.05 , por tanto, se acepta la hipótesis alterna $\left(\mathrm{H}_{1}\right)$ que afirma que existe correlación entre los puntajes obtenidos del desempeño del docente y los puntajes de las funciones de facilitador, investigador y promotor del logro del perfil de los egresados del semestre académico 2018 - II, del Programa de Educación Primaria de la Universidad Nacional del AltiplanoPuno.

Los resultados de la investigación dan a conocer que la correlación es directa y positiva entre el desempeño del docente y el perfil de logro del egresado del Programa de Educación Primaria de la Universidad Nacional del Altiplano - Puno, 2019, lo que significa que mientras con mayor satisfacción se encuentren los estudiantes en la evaluación el desempeño, mayor es el logro de competencias de los egresados en sus funciones de facilitador, investigador y promotor.

\section{Discusión}

Los autores manifiestan que el desempeño docente está vinculado con el logro de los aprendizajes de los estudiantes. Lourerio, Miguez y Otegui (2016) sustentan que la evaluación docente abarque diferentes variables que influyen en el proceso educativo, y que la información recogida sirve de insumo para tomar decisiones considerando el carácter formativo. Los estudiantes de la Facultad de Ingeniería de la Universidad de la República de Uruguay, consideran que los docentes obtienen calificaciones altas en motivación e interacción y puntualidad; así como en el dominio del tema (Bonilla, 2010). En esta misma perspectiva, Piña (2010) considera que existe una relación muy significativa entre desempeño docente, habilidades de estudiantes y el rendimiento académico en la Universidad Particular de Iquitos.

Referente al perfil de egreso de programas de estudio, Olivo, Voisin y Fernández (2015) consideran que los egresados demandan, entre otros, relaciones 
públicas y comunicación, y el manejo de marketing, lo que implicaría la evaluación del plan curricular. Los cambios que ocurren en los mercados de trabajo hacen necesarios que los estudiantes de la Licenciatura de Química Farmacéutica Bióloga de la Universidad Veracruzana, incorporen competencias que les permitan adaptarse permanente al cambio $\mathrm{y}$, además, se formen ciudadanos comprometidos (Martínez, 2015).

Considerando el desempeño docente y el logro de competencias del perfil profesional en los estudiantes del Instituto Túpac Amaru de Tinta Canchis, 2018 (considerando que es el logro del perfil de egreso), García y Zegarra (2018) muestran el valor de 0.830 según el estadístico de prueba para un estudio no paramétrico RHO de Spearman, lo que significa que existe una correlación alta y directa entre el desempeño docente en el aula y el logro de las competencias del perfil profesional.

Del mismo modo, Laura (2016) logró establecer el valor de 0,856, considerando que existe una relación directa y significativa entre el desarrollo curricular y el logro de competencias profesionales en estudiantes de la promoción 2013 de Telecomunicaciones e informática de la Universidad Nacional de Educación Enrique Guzmán y Valle, 2016. Guevara y Tupa (2015), concluye que existe relación entre el desempeño de los docentes y la competencia para la participación en la comunidad empresarial, de esto se deriva que los docentes vinculan a los alumnos a la comunidad empresarial.

En la misma línea de investigación, Ramírez (2009) muestra resultados donde el plan de estudios viene influenciando en el nivel de calidad de la formación profesional de los estudiantes de educación artística del ESPMI "Lorenzo Luján Darjón" y que los desempeños docentes en el aula influyen en la calidad de la formación profesional de los estudiantes de educación artística.

Finalmente, los resultados obtenidos del coeficiente de correlación de Spearman demuestran que el desempeño docente tiene una relación positiva muy alta cuyo valor de Rho $=0,849$ con el perfil de logro del egresado, además se tiene un coeficiente de determinación $\mathrm{R}^{2}=0,703$ que nos da conocer que aproximadamente el 70,3\% de la variación de la variable del perfil de logro del egresado puede ser explicada por la variación de la variable desempeño docente en el modelo. Asimismo, la ecuación de la regresión es: logegres $=8,60+1,23 *$ desdoc lo que da conocer que al aumento de una unidad de puntaje en el desempeño docente aumentará en 1,23 puntos, en el perfil de logro del egresado. Finalmente, la prueba de hipótesis de la t calculada $=6,711$ es mayor al $\mathrm{t}$ tabulada $=2,080$ confirmando lo establecido a un nivel de significancia del 5\%.

\section{CONCLUSIONES}

El desempeño docente está asociado al logro del perfil del egreso de manera positiva y muy alta. Además, en cuanto sea mejor el desempeño de los docentes, los estudiantes lograrán los rasgos previstos en el perfil del egreso de manera satisfactoria. El fortalecimiento de las competencias profesionales de los docentes de un programa de estudios universitarios tendría repercusión favorable en la formación de los profesionales.

Las funciones facilitador, investigador $y$ promotor del perfil del egreso son influenciados por el desempeño de los docentes. Esta valoración implica que los docentes al fortalecer 
las competencias profesionales relacionadas con la formación académica, investigación y responsabilidad social universitaria, favorecen de manera directa en la formación profesional de los estudiantes universitarios.

Los resultados de la evaluación del logro del perfil del egreso promueven la implementación de planes para mejorar los componentes curriculares; así como la de optimizar los procesos de aprendizaje y enseñanza, con fines de garantizar el logro de las competencias generales y específicos previstas en el currículo universitario.

\section{REFERENCIAS}

Barrientos, E. (2013) Las características de los docentes universitarios. Investigación Educativa Vol. 17, N² 2, 105-120

Benito, A. y Cruz, A. (2005). Nuevas Claves para la Docencia Universitaria. Madrid: Narcea. p. 19

Bonilla, J. (2010). Nivel de competencia y desempeño docente del profesorado de la escuela de medicina de la Universidad Católica Nordestana (UCNE) bajo un enfoque integral y desarrollador. Universidad del País Vasco Euskal Herriko Unibertsitatea-España. 2010

Carrizales, C. y Rodríguez, L. (1999). La formación profesional del docente universitario. Rev. Cubana Educ. Med. Sup. 13 (1), 80-91

Cruz, M. (2007). Una propuesta para la evaluación del profesorado universitario. Tesis doctoral inédita. Universidad de Barcelona

Echeverría, B. (2002). Gestión de la competencia de acción profesional. Revista de Investigación Educativa, 20(1), 7-43

Feeney, S. (2001): El campo del curriculum en la Argentina. Un análisis de los discursos que acerca del curriculum producen los pedagogos argentinos: 1983-1998. Tesis de Maestría en Didáctica. Facultad de Filosofía y Letras. Universidad Nacional de Buenos Aires

Fernández, A. (2010). La evaluación orientada al aprendizaje en un modelo de formación por competencias en la educación universitaria. Vol.8 (n.1) 11-34. Recuperado de http://red-u. net/redu/documentos/vol8_n1_completo.pdf

García, C. y Zegarra, W. (2018). Desempeño docente y logro de competencias del Instituto Tupac Amarú de Tinta - Canchis 2018. Universidad César Vallejo, Trujillo

Guevara, A. y Tupa, L. (2015). Análisis de la relación entre las competencias deseadas y el desempeño del docente universitario. Tesis de Pre Grado. Universidad Nacional de Huamanga, Ayacucho

Gutiérrez, H., (2018). La Evaluación desde el Enfoque Socioformativo: Ejes y Herramientas. En S. R. Herrera-Meza y S. Tobón (Coords.), Memorias del III Congreso Internacional de Evaluación (Valora-2018). México: Centro Universitario CIFE

Huanca, J. N. (2017). La Evaluación de Competencias desde el Enfoque Socioformativo México: Centro Universitario MARCO

Hernández-Sampieri, R. \& Mendoza, C (2018). Metodología de la investigación. Las rutas cuantitativa, cualitativa y mixta, Ciudad de México, México: Editorial Mc Graw Hill Education, Año de edición: 2018, ISBN: 978-14562-6096-5, $714 \mathrm{p}$

Irigoin, M. (2006). Desafíos de la formación por competencias en la educación superior. El informativo de la educación superior, 318. Extraído el 5 de Enero de 2007 desde http:// www.mecesuo.cl/informativo/paginas/ cuerpo.pep?ided $i=2006071431107 \&$ idele $=20060714204742$

Laura, A. (2016). Desarrollo curricular y el logro de competencias profesionales en estudiantes de Telecomunicaciones e Informática de la Universidad Nacional de Educación Enrique Guzmán y Valle, 2016. Universidad Nacional Enrique Guzmán y Valle, la Cantuta, Lima

Le Boterf, G. (2001). Ingeniería de las competencias. Barcelona: Gedisa

Ley Universitaria Peruana $N^{\circ} 30220$ (2014). Recuperado en fecha: 09/07/2014 en https://www.sunedu.gob.pe/nueva-leyuniversitaria-30220-2014/ 
Lourerio, S., Miguez, M. y Otegui X. (2016) Desempeño docente en la enseñanza universitaria: análisis de las opiniones estudiantiles. Cuadernos de Investigación Educativa, Vol. 7, № 1, 2016, Montevideo (Uruguay), 55-67. Universidad ORT Uruguay. DOI: http://dx.doi.org/10.18861/ cied.2016.7.1.2576

Loureiro, S. y Míguez, M. (2006) Evaluación docente. La experiencia en la Facultad de Ingeniería de la Universidad de la República. Revista Iberoamericana de Educación. [Revista electrónica], 40 (5). Disponible en: http:// www.rieoei.org/experiencias137.htm [Fecha de consulta: octubre 19 de 2015]

Martínez, L. (2015). Evaluación del perfil de egreso: primer paso para la reformulación del currículum. CPU-e, Revista de Investigación Educativa, (21), 210-221

Mateo, J. (2000). "La evaluación educativa, su práctica y otras metáforas" Editorial Horsori Barcelona España

Mateo, M., Escudero, T., De Miguel, M., Ginés, J. y Rodríguez, S. (1996) La evaluación del profesorado. Un tema a debate. Revista de Investigación Educativa, 14(2), 73-94

Muñiz, J., García, A. y Virgos, J. (1991) Escala de la Universidad de Oviedo para la evaluación del profesorado. Psicothema 3(2), 269-281

Muñoz, J. M.; Ríos, M. P. y Abalde, E. (2002) Evaluación Docente vs. Evaluación de la Calidad. Revista Electrónica de Investigación y Evaluación Educativa (RELIEVE). [Revista electrónica], 8, (2), Disponible en: http://www. uv.es/RELIEVE/v8n2/RELIEVEv8n2_4. htm [Fecha de consulta: octubre 19 de 2015]

Olivos, M., Voisin, S., y Fernández, J. (2015). Evaluación del perfil de egreso de profesores de francés de parte de los empleadores: propuestas de mejora y desarrollo. Revista Electrónica "Actualidades Investigativas en Educación", 15 (1), 1-16

Piña, R. (2010). El desempeño docente y su relación conlas habilidadesdel estudianteyel rendimiento académico en la Universidad Particular de
Iquitos, año 2010. Universidad Mayor de San Marcos. Recuperado de http://cybertesis. unmsm.edu.pe/handle/cybertesis/2366

Quesada, R. (2001) Cómo plantear la Docencia Estratégica. México. Limusa

Ramírez, V. (2009). El Plan de estudios, desempeño docente, los recursos tecnológicos y la calidad de la formación profesional de los estudiantes de Educación Artística de la Escuela Superior Pública de Música Lorenzo Luján Darjón de Iquitos en el año 2008. Universidad Nacional Mayor de San Marcos. Recuperado de http://cybertesis.unmsm.edu. pe/bitstream/handle/cybertesis/2391/Ramirez_ gh.pdf? sequence $=1 \&$ isAllowed $=y$

Rebolledo, M. A., y Ayala, M. (2006). Evaluación del desempeño docente del Departamento de Biología Marina de UABCS. Revista Latinoamericana de estudios educativos, 36 (12), 95-133

Sistema Nacional de Evaluación, Acreditación y Certificación de la Calidad Educativa. (2016). Modelo de Acreditación para Programas de Estudios de Educación Superior Universitaria. Lima

Stiggins, R.J., Duke, D.L. (1988). The Case for Commitment to Teacher Growth: Research on Teacher Evaluation, State University of New York. Press, Albany, NY

Tobón, S. (2018). Metodología de la evaluación socioformativa. En S. R. Herrera-Meza y S. Tobón (Coords.), Memorias del III Congreso Internacional de Evaluación (Valora-2018). México: Centro Universitario CIFE

Tobón, S. (2017). Evaluación socioformativa. Estrategias e instrumentos. Mount Dora (USA): Kresearch

Tobón, S. (2015). La Evaluación Socioformativa Estrategias e instrumentos. Estados Unidos: CIFE Corporation

Tobón, S., González, L., Nambo, JM. y Vázquez, JM., (2015). La Socioformación: Un Estudio Conceptual, Paradigma, Vol, XXXVI (1), 7-29. Recuperado de http://revistas.upel.edu.ve/ index.php/paradigma/article/view/2661 
Tobón, S. (2006). Competencias, calidad y educación superior. Bogotá: Magisterio

Tobón (2005). Formación Basada en Competencias. 2da edición: Ecoe Ediciones. Bogotá

Universidad Nacional del Altiplano. Puno. (2018). Reglamento de evaluación del desempeño docente. Puno
Valdés, H. (2000). Evaluación del Desempeño del Docente en Cuba. Ponencia presentada en Encuentro Iberoamericano sobre Evaluación del Desempeño Docente. Ciudad de México, 23 al 25 de mayo de 2000

Zabalza, Miguel. (2003). Competencias docentes del profesorado universitario. Calidad y desarrollo profesional. Narcea, España 